\title{
Hortiterapia melhora as habilidades sociais e de comunicação de jovens portadores de necessidades especiais ${ }^{(1)}$
}

\author{
MARCELA MAGALY MARÇALLO ${ }^{(2)}$, MARIA CAROLINA SABBAGH ${ }^{(3)}$ e FRANCINE LORENA CUQUEL ${ }^{(4)}$
}

\section{RESUMO}

A apreciação passiva da natureza, assim como a participação ativa no jardim cultivando plantas influi positivamente nas emoções, na saúde e no comportamento das pessoas. A Hortiterapia é uma técnica que permite às pessoas apresentem maior bem-estar físico e mental por meio do cultivo das plantas e do contato com o ambiente. As atividades de Hortiterapia podem ser executadas por pessoas portadoras de necessidades especiais (PPNE), por pacientes geriátricos e por indivíduos sob risco, como viciados em drogas e álcool, influenciando positivamente na saúde, na emoção e no comportamento desses indivíduos. O objetivo desta pesquisa foi avaliar o desenvolvimento das habilidades sociais e da comunicação de PPNE — com deficiência mental moderada — submetidas ao Programa de Hortiterapia. Esse Programa foi aplicado na Fundação Ecumênica de Proteção ao Excepcional (FEPE) para 24 jovens de ambos os sexos (com idade entre 20 e 25 anos), PPNE com deficiência mental moderada. Eles foram organizados em três grupos com oito participantes cada, e cada grupo foi submetido a um Programa de Hortiterapia com duração de uma hora por semana, durante 20 semanas. Antes e depois das atividades de Hortiterapia, os participantes foram submetidos a Teste Palográfico (ALVES e ESTEVES, 2004), teste este que permite avaliar capacidade de organização, emotividade, depressão e impulsividade. Durante todo o programa, foram avaliados a habilidade social, a adaptabilidade, os contatos visuais e físicos, a expressão facial, as habilidades de organização, de comunicação e comportamental. Ao término do Programa verificou-se que as PPNE submetidas à Hortiterapia tiveram melhoria em, pelo menos, uma das habilidades avaliadas e que, freqüentemente, mais de uma das habilidades avaliadas melhoraram simultaneamente.

Palavras-chave: horticultura, jardinagem, deficiência mental, deficiência intelectual, educação especial, programa educacional, atividade recreativa, psicologia.

\begin{abstract}
Hortitherapy improves the social and communication skills of youths with moderate mental deficiency

The passive appreciation of the nature, as well as the active participation in the garden, influences positively emotions, health and people behavior. Hortitherapy is a technique that allows mental and physical welfare by cultivating plants and through the contact with nature. The activities of Hortitherapy can be applied to people with mental and physical deficiencies, geriatric patients and individuals under risk, as drugs and alcohol dependents, influencing positively the health, emotion and behavior of these individuals. The goal of this research was to evaluate the social abilities and the communication of young individuals with mental deficiency, submitted to a Program of Hortitherapy. This Program was applied in the Ecumenical Foundation of Protection of Excepcionals (FEPE), for 24 young men and women (with 20 to 25 years old) with moderate mental deficiency. They had been organized in three groups of eight participants each one, and each group was submitted to a Program of Hortitherapy with duration of one hour per week during 20 weeks. Before and after the activities of Hortitherapy the participants were submitted to a test (ALVES and ESTEVES, 2004) that allows the evaluation of the organization capacity, emotion, depression and impulsiveness. During the Program they had been evaluated as to social ability, visual possible to verify an improvement in at least one variable of the evaluation criteria, and frequently other variables were simultaneously improved.
\end{abstract}

Key words: horticulture, gardening, mentally retarded, intellectual disability, special-education, educational programs, recreational activity, psychology.

\footnotetext{
(1) Recebido para publicação em 25/05/2006 e aceito em 14/10/2007.

(2) Psicóloga - marcelamarcallo@ hotmail.com

${ }^{(3)}$ Engenheira Agrônoma e Mestranda de Agronomia - Produção Vegetal - carol.sabbagh@terra.com.br

(4) Professora da Universidade Federal do Paraná - francine@ufpr.br
} 


\section{INTRODUÇÃO}

Pessoas portadoras de necessidades especiais (PPNE) têm necessidades específicas resultantes da perda ou anomalia de uma estrutura ou função psicológica, fisiológica ou anatômica(ORGANIZAÇÃO MUNDIALDA SAÚDE, 2000). Aproximadamente, $10 \%$ da população dos países em desenvolvimento são portadores de algum tipo de necessidade especial (ORGANIZAÇÃO MUNDIAL DA SAÚDE, 2000). O censo do IBGE de 2005 (FUNDAÇÃO INSTITUTOBRASILEIROGEOGRAFIAEESTATÍSTICA, 2005) apontou que a população do Brasil se aproxima de 170 milhões de habitantes; logo, estima-se que 17 milhões de brasileiros possuam algum tipo de deficiência. Desse total, $50 \%$ são portadores de deficiência mental.

PPNE apresentam dificuldades de adaptação às exigências culturais da sociedade em que estão inseridas, dificuldades estas que se acentuam à medida em que forem mais significativas as suas deficiências, tanto no plano cognitivo quanto adaptativo (EVANGELISTA, 2002). O objetivo principal do atendimento a PPNE é direcioná-las para a habilitação, visando à melhoria dos comportamentos adaptativos para possibilitar uma integração na vida comunitária (DEL PRETTE e DEL PRETTE, 2002).

A apreciação passiva da natureza, bem como a participação ativa no jardim, cultivando as plantas, influi positivamente nas emoções, na saúde e no comportamento das pessoas (RELF, 1981). A Hortiterapia é uma técnica que permite às pessoas alcancem maior bem-estar físico $\mathrm{e}$ mental por meio do cultivo das plantas e do contato com a natureza (RELF, 1992). As atividades de Hortiterapia podem ser aplicadas a PPNE, a pacientes geriátricos e a indivíduos sob risco, como viciados em drogas e álcool, influenciando positivamente a saúde, a emoção e o comportamento desses indivíduos (FILIPSKI, 2005).

A Hortiterapia vem sendo aplicada com bons resultados no Canadá, na Nova Zelândia, nos Estados Unidos e na Europa. Pesquisas feitas com PPNE mostraram que a Hortiterapia favorece a inclusão, respeitando as capacidades de aprendizado de cada um, valorizando conhecimentos, respeitando limitações, reconhecendo diferenças e ressaltando potencialidades (HEFLEY,1973). Com base na integração homem-planta (por meio da manipulação e do aprendizado de técnicas para a produção de plantas), as PPNE têm a oportunidade de trabalhar vários aspectos, tais como: a manipulação de plantas conhecidas em seu dia-a-dia, a integração e a reintegração dessas pessoas no meio social, a elevação da auto-estima, o poder de concentração, a estipulação de regras e horários, o estímulo do reconhecimento do seu trabalho, a sua própria valorização e o favorecimento da comunicação (GILLIES,2006).

O objetivo desta pesquisa foi avaliar o efeito de um Programa de Hortiterapia a PPNE, com deficiência mental moderada, inseridas no programa Préprofissionalizante da Fundação Ecumênica de Proteção ao Excepcional (FEPE), na cidade de Curitiba (Paraná), Brasil.

\section{MATERIALEMÉTODOS}

O programa de Hortiterapia foi aplicado a jovens de ambos os sexos na faixa etária de 20-25 anos, os quais estavam matriculados na FEPE, uma Fundação de apoio à comunidade de Curitiba (Paraná). Os jovens participantes do Programa de Hortiterapia foram pré-selecionados pela FEPE, de acordo com o nível de QI, de 25 a 75, pela autonomia parcial em AVD'S (Atividades da Vida Diária). Esses jovens executam sua higiene pessoal e têm autonomia para se vestirem e se alimentarem, além de demonstrarem cuidados com seus pertences e capacidade de compreensão, respondendo a ordens simples com linguagem que possibilitava as comunicações verbais, gestuais e leitura funcional. Face a essas habilidades, eles estavam matriculados no Programa Pré-Profissionalizante dessa Fundação. Outra premissa para a seleção foi que esses jovens não tivessem tido nenhum contato anterior com atividades hortícolas, no que tange a informação sobre plantas e cultivo. Os pais e/ou representantes legais dos participantes foram informados do propósito do estudo e autorizaram a inclusão deles no Programa.

Antes do início do Programa, foram analisadas fichas, elaboradas pela FEPE, contendo o histórico de cada jovem selecionado, e fichas de hábitos de trabalhos, diariamente preenchidas pelos participantes. Além disso, foram entrevistados os profissionais que trabalhavam com os jovens. Finalmente, foram efetuadas entrevistas semiestruturadas com cada participante.

No início e no final do Programa, foi aplicada a Escala de Autoconceito Infanto-Juvenil (SISTO e MARTINELLI, 2004). Essa escala permite avaliar o autoconceito nos contextos social, escolar, familiar e pessoal, medindo também a qualidade das relações que as pessoas estabelecem consigo e com as outras. Além disso, foi aplicado o Teste Palográfico (ALVES e ESTEVES, 2004). Esse teste baseia-se em uma técnica gráfica que envolve a elaboração de traçados simples, com uma variedade quase ilimitada de respostas possíveis, que foram analisadas por meio de padrões comparativos padronizados (ALVES e ESTEVES, 2004). Usando esse teste, foi possível avaliar a adaptação, a expressão e a projeção da personalidade, com os seguintes aspectos: organização (avalia a forma de pensar e agir, de acordo com o plano, método ou regra de maneira clara), a emotividade (caracteriza-se por vibrações, choques ou comoções interiores das funções psicológicas e fisiológicas), a depressão (avalia a queda brusca da tensão neuro-muscular com redução da atividade física e psíquica), e a impulsividade (avalia a capacidade de conter reações impulsivas).

Os participantes foram organizados em três grupos homogêneos, cada um com oito jovens de ambos os sexos. Esses jovens participaram do Programa de Hortiterapia por um período de vinte semanas, em encontros com duração de noventa minutos, que ocorriam semanalmente.

Todo o Programa foi desenvolvido nas dependências da própria Fundação, com atividades 
externas e internas. Nas atividades externas, priorizando o contato físico com a natureza e com as plantas, foram desenvolvidas atividades de horticultura, a partir da semeadura de espécies que tivessem diferentes tamanhos e formas de sementes, folhas e fins, e aplicação das técnicas básicas de cultivo, com substrato em embalagens. As espécies escolhidas foram divididas em três grupos: Grupo 1- plantas agrícolas (Zea mays, Phaseolus vulgaris, Araucaria angustifolia); Grupo 2- plantas medicinais (Matricaria chamomilla, Majerona hortensis, Foeniculum vulgare); Grupo 3- plantas ornamentais (Dianthus chinensis, Helianthus annuus, Viola tricolor).

Foram disponibilizadas 45 unidades por participante (sementes ou embalagens), as quais foram semeadas sob monitoria, explicando-lhes a importância de que eles lhes dessem os cuidados necessários. Entre os cuidados, estava a irrigação e o raleio de plantas daninhas. Nas atividades internas, foram abordados temas relacionados com as plantas e com a natureza, tais como educação ambiental, a importância da água e poluição. Em todos os encontros, foram feitas correlações entre as espécies que haviam sido semeadas e o uso delas na rotina de cada participante, por meio de aromas, sabores e texturas. Também foram utilizados recursos visuais, audiovisuais e auditivos, como apresentação de livros didáticos e revistas contendo fotos temáticas, filmes temáticos e sons da natureza, além de jogos de memória, verbalizações das atividades externas, finalizando com a fixação dos temas, quando os participantes fizeram desenhos representativos.

\section{RESULTADOS E DISCUSSÂO}

Ao final do Programa de Hortiterapia, as PPNE melhoraram no aspecto organização (Tabela 1). O trabalho com plantas proporcionou melhoria significativa na elaboração de esquemas e no aumento do senso de responsabilidade. Isso concorda com os resultados obtidos num Programa de Hortiterapia aplicado no Hospital Psiquiátrico de Kansas/USA, em que mais da metade dos participantes aumentaram seu senso de responsabilidade durante o Programa, o que foi demonstrado pela manutenção das plantas que estavam sob os cuidados dos indivíduos (SELLERS, 2001). Comparando os três grupos, pode-se dizer que, no grupo 1, o aumento da organização esteve presente em 14,8\% dos participantes; no grupo 2 , o aumento foi de $37,5 \%$, e no grupo 3 , foi de $37,5 \%$, totalizando uma média de $29,76 \%$ de melhoria nos três grupos.

Da mesma forma, as PPNE melhoraram no aspecto emotividade (Tabela 2). Isso, provavelmente, ocorreu porque, conforme citou BLANCO (2005), pessoas com problemas de comunicação, quando em contato com plantas e jardins, aprendem a expressar seus sentimentos e a estabelecer relações interpessoais. Segundo GILLIES (2006), a manipulação de plantas conhecidas em seu dia-adia promove a elevação da auto-estima. Já para RELF (2004), pessoas que tiveram contato com Hortiterapia diminuíram os sentimentos de raiva e agressividade. Constatou-se que, no grupo $1,57,1 \%$ dos participantes obtiveram melhoria no aspecto emocional; no grupo 2, 37,5\%, e no grupo 3, a melhoria se deu em $37,5 \%$ dos participantes, totalizando uma média de 44,03\% de melhoria nos grupos avaliados.

Constatou-se que a depressão diminuiu em alguns indivíduos submetidos ao Programa (Tabela 3). Isso, provavelmente, ocorreu porque o contato com plantas, que necessitam de cuidado e atenção, cria responsabilidade no indivíduo, fazendo-o sentir-se útil e importante, combatendo, desta forma, alguns dos sintomas de depressão. Como os exercícios físicos aliados aos constantes estímulos e contatos com sabores, aromas, texturas e sons estimulam a produção de endorfina - que é o hormônio relacionado com a sensação de bem-estar geral (AMERICAN PSYCHIATRIC ASSOCIATION, 1995) talvez, também possa ter ajudado a reduzir a depressão. Em alguns participantes com esses sintomas, constatouse uma melhora de $100 \%$.

As PPNE reduziram a sua impulsividade (Tabela 4). Estudos mostram que as pessoas que convivem por períodos prolongados com plantas apresentam condutas e ações mais estabilizadas. De acordo com KUO e SULLIVAN (2001), em pesquisa com indivíduos residentes na periferia e com comportamento agressivo e violento, esse comportamento foi reduzido quando eles permaneceram maior tempo em contato com árvores e um amplo gramado. No presente trabalho, observou-se que, no grupo 1, houve uma melhoria em $85,7 \%$ dos participantes; no grupo 2, a melhoria foi de $2,5 \%$, e no grupo 3 , foi de $37,5 \%$, totalizando uma média de $42,5 \%$. de melhoria nos grupos avaliados.

Considera-se, dos três grupos avaliados, o grupo 1 o que obteve melhores resultados nos aspectos organizacional, emocional e impulsividade. Esses resultados, provavelmente, ocorreram pelo grau de informações que os pais e/ou responsáveis têm a respeito das limitações dos participantes, provendo a eles o estímulo necessário para que possam se desenvolver; pela interação entre si e com as pesquisadoras desse Programa e pelo interesse em aprender assuntos novos, constatados nas entrevistas feitas.

No aspecto depressão, o grupo que obteve resultados positivos foi o 2 . Esse fato leva a crer que, mesmo com a dificuldade motora e com a falta de interação entre si, os indivíduos tiveram um aumento em sua autoestima, sentindo-se úteis com o estímulo do reconhecimento do seu trabalho e, ao final, tiveram maior contato físico com as pesquisadoras do Programa.

Além dos aspectos anteriormente abordados, os participantes também tiveram, ao final do Programa, maior capacidade de verbalização, maior interação com o grupo, maior exteriorização dos seus sentimentos, incremento no contato visual, maior contato físico e maior capacidade de expressão facial.

Esses resultados demonstram a possibilidade de utilização de Hortiterapia como uma das ferramentas para incremento dos comportamentos adaptativos, o que pode favorecer a inclusão social de jovens portadores de deficiências mentais. 


\section{CONCLUSÂO}

O Programa de Hortiterapia permitiu que PPNE apresentassem melhorias significativas na capacidade de organização, emotividade mais controlada, menor depressão e menor impulsividade.

\section{REFERÊNCIAS}

ALVES, I.C.B.; ESTEVES C. O Teste Palográfico na avaliação da personalidade. ed. São Paulo: Vetor Editora, 2004. $196 \mathrm{p}$.

AMERICAN PSYCHIATRIC ASSOCIATION. Manual de diagnóstico e estatística de transtornos mentais (DSM IV). 4. ed. Porto Alegre: Ed. Rev. Artes Médicas, 1995.

BLANCO, L. M. Terapia hortícola: una técnica que cura a los enfermos en el jardín. Disponível em:

http://www.el-mundo.es. Acesso em: 20 jul.2005.

DEL PRETTE, Z; DEL PRETTE, A. Psicologia das habilidades sociais: terapia e educação. 3. ed. Rio de Janeiro: Vozes, 2002. 89p.

EVANGELISTA, L. M. C. Novas abordagens do diagnóstico psicológico da deficiência mental. ed. São Paulo: Vetor Editora Psico-Pedagógica, 2002. 260 p.

FILIPSKI J. Hortiterapia. Disponível em: http://www.gardenforever.com. Acessado em: 30 mar. 2005.
FUNDAÇÃOINSTITUTO BRASILEIRO GEOGRAFIAE ESTATÍSTICA. Disponível em: 〈www.ibge.gov.br〉. Acesso em: 17 jun. 2005.

GILLIES, B. Why Horticulture therapy? Disponível em: www.greenweb.com.au. Acesso em: 22 fev.2006.

HEFLEY P.D. Horticulture: a therapeutic tool. Journal of Rehabilitation, Alexandria, v.39, n.1, p.27-29. 1973.

KUO, F.E.; SULLIVAN, W. C. Aggression and violence in the inner city. Environment and Behavior, Beverly Hills, v.33, n.4, p.543-571, 2001.

ORGANIZAÇÃO MUNDIALDA SAÚDE. Manejo de desnutrição grave: manual para profissionais de saúde de nível superior. Genebra, 2000.76p.

RELF, P.D. Human issues in Horticulture. Hort Technology, Alexandria v.2, p.159-170, 1992.

The evolution of the People-Plant Council: an assessment of the first twelve years In: XXVI Congresso de Horticultura Internacional, Toronto, 2004. 1 CDROM.

The use of horticulture in vocational rehabilitation. Journal of Rehabilitation, Alexandria, v.47, n.3, p.53-60, 1981.

SELLERS, K.D. Effectiveness of horticultural therapy activities in a psychiatric hospital. Journal of Therapeutic Horticulture, Austin, v. 12, p.9-20, 2001.

SISTO, F.F.; MARTINELLI, S.C. (Ed.). Escala de autoconceito infanto-juvenil. ed. São Paulo: Vetor Editora Psico-Pedagógica Ltda., 2004. 88 p. 
Tabela 1. Avaliação organizacional, obtida com o Teste Palográfico, de 24 PPNE (20 a 25 anos) após Programa de Hortiterapia durante 20 semanas. Curitiba (PR). 2005

\begin{tabular}{cllclllll}
\hline Grupo 1 & Inicial & Final & Grupo 2 & Inicial & Final & Grupo 3 & Inicial & Final \\
\hline 1 & Ruim & Ruim & 1 & Muito ruim & Muito ruim & 1 & Muito ruim & Ruim \\
2 & Ruim & Regular & 2 & Ruim & Regular & 2 & Ruim & Ruim \\
3 & Ruim & Ruim & 3 & Muito ruim & Muito ruim & 3 & Ruim & Regular \\
4 & Ruim & Ruim & 4 & Muito ruim & Muito ruim & 4 & Ruim & Regular \\
5 & Ruim & Ruim & 5 & Muito ruim & Muito ruim & 5 & Regular & Regular \\
6 & Muito ruim & Muito Ruim & 6 & Muito ruim & Muito ruim & 6 & Ruim & Ruim \\
7 & Ruim & Ruim & 7 & Regular & Boa & 7 & Ruim & Ruim \\
$8 * * *$ & Regular & & $-\ldots$ & Muito ruim & Regular & 8 & Ruim & Ruim \\
\hline
\end{tabular}

$* * *$ desistiu na $3^{\mathrm{a}}$ semana do projeto.

Muito Ruim = ausência de qualidade na execução das atividades.

Ruim = com baixa qualidade na execução das atividades.

Regular = qualidade regular na execução das atividades

Boa = boa qualidade de execução das atividades.

Table 1. Organizational Capacity Evaluation by Palographic Test, applied to 24 patients with moderate mental deficiency (20 to 25 years) after Hortitherapy Program during 20 weeks. Curitiba (PR). 2005.

\begin{tabular}{|c|c|c|c|c|c|c|c|c|}
\hline Group 1 & First & Last & Group 2 & First & Last & Group 3 & First & Last \\
\hline 1 & $\mathrm{Bad}$ & $\mathrm{Bad}$ & 1 & Worse & Worse & 1 & Worse & Bad \\
\hline 2 & $\mathrm{Bad}$ & Regular & 2 & Bad & Regular & 2 & $\mathrm{Bad}$ & $\mathrm{Bad}$ \\
\hline 3 & Bad & $\mathrm{Bad}$ & 3 & Worse & Worse & 3 & Bad & Regular \\
\hline 4 & $\mathrm{Bad}$ & Bad & 4 & Worse & Worse & 4 & Bad & Regular \\
\hline 5 & $\mathrm{Bad}$ & $\mathrm{Bad}$ & 5 & Worse & Worse & 5 & Regular & Regular \\
\hline 6 & Worse & Worse & 6 & Worse & Worse & 6 & $\mathrm{Bad}$ & $\mathrm{Bad}$ \\
\hline 7 & $\mathrm{Bad}$ & $\mathrm{Bad}$ & 7 & Regular & Good & 7 & $\mathrm{Bad}$ & $\mathrm{Bad}$ \\
\hline $8 * * *$ & Regular & --- & 8 & Worse & $\mathrm{Bad}$ & 8 & $\mathrm{Bad}$ & $\mathrm{Bad}$ \\
\hline $\begin{array}{l}* * *=\mathrm{A} \\
\text { First = F } \\
\text { Lst = La } \\
\text { Worse = } \\
\text { Bad = A } \\
\text { Regular } \\
\text { Good = }\end{array}$ & $\begin{array}{l}\text { bandone } \\
\text { First Eva } \\
\text { ast evalu } \\
=\text { Activiti } \\
\text { Activities } \\
=\text { Activit } \\
\text { Activitie }\end{array}$ & $\begin{array}{l}\text { rogram afte } \\
\text { rmed with } \\
\text { hed with lov } \\
\text { formed with } \\
\text { rmed with g }\end{array}$ & $\begin{array}{l}\text { of quality } \\
\text { lity. } \\
\text { lar qualit } \\
\text { quality. }\end{array}$ & $\begin{array}{l}\text { y. } \\
\text { ty. }\end{array}$ & & & & \\
\hline
\end{tabular}

Tabela 2. Avaliação emocional, obtida com o Teste Palográfico, em 24 PPNE (20 a 25 anos) após Programa de Hortiterapia durante 20 semanas. Curitiba (PR). 2005

\begin{tabular}{|c|c|c|c|c|c|c|c|c|}
\hline Grupo 1 & Inicial & Final & Grupo 2 & Inicial & Final & Grupo 3 & Inicial & Final \\
\hline 1 & Média & Média & 1 & Muito aum. & Aumentada & 1 & Média & Média \\
\hline 2 & Média & Média & 2 & Média & Média & 2 & Média & Média \\
\hline 3 & Diminuída & Média & 3 & Aumentada & Aumentada & 3 & Média & Média \\
\hline 4 & Diminuída & Média & 4 & Diminuída & Média & 4 & Diminuída & Diminuída \\
\hline 5 & Média & Média & 5 & Aumentada & Média & 5 & Diminuída & Diminuída \\
\hline 6 & Aumentada & Média & 6 & Média & Média & 6 & Diminuída & Média \\
\hline 7 & Aumentada & Média & 7 & Diminuída & Diminuída & 7 & Aumentada & Média \\
\hline $8 * * *$ & Diminuída & --- & 8 & Média & Média & 8 & Muito aum. & Aumentada \\
\hline
\end{tabular}

$* * *=$ desistiu na $3^{\mathrm{a}}$ semana do projeto.

Muito aum. e Aumentada= choques e mudanças de ânimo violento.

Média $=$ emotividade ideal.

Diminuída = choques lentos, retardados e graves, levando à apatia, indiferença e indolência afetiva.

Table 2. Emotional Evaluation by Palographic Test, applied to 24 patients with moderate mental deficiency (20 to 25 years) after Hortitherapy Program during 20 weeks. Curitiba (PR). 2005.

\begin{tabular}{cllclllll}
\hline Group 1 & First & Last & Group 2 & First & Last & Group 3 & First & Last \\
\hline 1 & Average & Average & 1 & Very High & High & 1 & Average & Average \\
2 & Average & Average & 2 & Average & Average & 2 & Average & Average \\
3 & Low & Average & 3 & High & High & 3 & Average & Average \\
4 & Low & Average & 4 & Low & Average & 4 & Low & Low \\
5 & Average & Average & 5 & High & Average & 5 & Low & Low \\
6 & High & Average & 6 & Average & Average & 6 & Low & Average \\
7 & High & Average & 7 & Low & Low & 7 & High & Average \\
$8 * * *$ & Low & --- & 8 & Average & Average & 8 & Very High High \\
\hline
\end{tabular}

$* * *=$ Abandoned the Program after the $3^{\text {rd }}$ week

Very high and high $=$ Clashes and violent changes of mind.

Media = Controlled emotional.

Low $=$ Shock slow, delayed and serious, leading to apathy, indifference and earnestness affective. 
Tabela 3. Avaliação da depressão, obtida com o Teste Palográfico, em 24 PPNE (20 a 25 anos) após Programa de Hortiterapia durante 20 semanas. Curitiba (PR). 2005

\begin{tabular}{|c|c|c|c|c|c|c|c|c|}
\hline Grupo 1 & Inicial & Final & Grupo 2 & Inicial & Final & Grupo 3 & Inicial & Final \\
\hline 1 & Não & Não & 1 & Sim & Sim & 1 & Não & $\overline{\text { Não }}$ \\
\hline 2 & Não & Não & 2 & Sim & Não & 2 & Não & Não \\
\hline 3 & Não & Não & 3 & Não & Não & 3 & Não & Não \\
\hline 4 & Não & Não & 4 & Não & Não & 4 & Não & Não \\
\hline 5 & Não & Não & 5 & Sim & Sim & 5 & Não & Não \\
\hline 6 & Não & Não & 6 & Não & Não & 6 & Não & Não \\
\hline 7 & Não & Não & 7 & Não & Não & 7 & Não & Não \\
\hline $8 * * *$ & Sim & --- & 8 & Sim & Não & 8 & Não & Não \\
\hline
\end{tabular}

Table 3. Depression Evaluation by Palographic Test, applied to 24 patients with moderate mental deficiency (20 to 25 years) after Hortitherapy Program during 20 weeks. Curitiba (PR). 2005.

\begin{tabular}{lllllllll}
\hline Group 1 & First & Last & Group 2 & First & Last & Group 3 & First & Last \\
\hline 1 & No & No & 1 & Yes & Yes & 1 & No & No \\
2 & No & No & 2 & Yes & No & 2 & No & No \\
3 & No & No & 3 & No & No & 3 & No & No \\
4 & No & No & 4 & No & No & 4 & No & No \\
5 & No & No & 5 & Yes & Yes & 5 & No & No \\
6 & No & No & 6 & No & No & 6 & No & No \\
7 & No & No & 7 & No & No & 7 & No & No \\
$8 * * *$ & Yes & --- & 8 & Yes & No & 8 & No & No \\
\hline$* * *=$ Abandoned the Program after 3 $3^{\text {rd }}$ week & & & \\
Yes = Depressed. & \\
No= Non Depressed.
\end{tabular}

Tabela 4. Avaliação da impulsividade, obtida com o Teste Palográfico, em 24 jovens (20 a 25 anos) PPNE após Programa de Hortiterapia durante 20 semanas. Curitiba (PR). 2005

\begin{tabular}{|c|c|c|c|c|c|c|c|c|}
\hline Grupo 1 & Inicial & Final & Grupo 2 & Inicial & Final & Grupo 3 & Inicial & Final \\
\hline 1 & Muito aum. & Aumentada & 1 & Muito aum. & Muito aum. & 1 & Aumentada & Aumentada \\
\hline 2 & Muito aum. & Média & 2 & Média & Média & 2 & Muito aum. & Aumentada \\
\hline 3 & Média & Média & 3 & Diminuída & Diminuída & 3 & Muito aum. & Aumentada \\
\hline 4 & Muito aum. & Média & 4 & Muito aum. & Muito aum. & 4 & Média & Média \\
\hline 5 & Muito aum. & Aumentada & 5 & Média Média & & 5 & Média & Média \\
\hline 6 & Muito aum. & Aumentada & 6 & Muito aum & Muito aum. & 6 & Muito aum. & Muito aum. \\
\hline 7 & Aumentada & Média & 7 & Diminuída & Média & 7 & Média & Média \\
\hline $8 * * *$ & Diminuída & --- & 8 & Muito aum & Média & 8 & Aumentada & Média \\
\hline
\end{tabular}

Table 4. Impulsiveness Evaluation by Palographic Test, applied to 24 patients with moderate mental deficiency (20 to 25 years) after Hortitherapy Program during 20 weeks. Curitiba (PR). 2005.

\begin{tabular}{|c|c|c|c|c|c|c|c|c|}
\hline Group 1 & First & Last & Group 2 & First & Last & Group 3 & First & Last \\
\hline 1 & Very High & High & 1 & Very High & Very High & 1 & High & High \\
\hline 2 & Very High & Average & 2 & Average & Average & 2 & Very High & High \\
\hline 3 & Average & Average & 3 & Low & Low & 3 & Very High & High \\
\hline 4 & Very High & Average & 4 & Very High & Very High & 4 & Average & Average \\
\hline 5 & Very High & High & 5 & Average & Average & 5 & Average & Average \\
\hline 6 & Very High & High & 6 & Very High & Very High & 6 & Very High & Very High \\
\hline 7 & High & Average & 7 & Low & Average & 7 & Average & Average \\
\hline $8 * * *$ & Low & --- & 8 & Very High & Average & 8 & High & Average \\
\hline
\end{tabular}

